\title{
TRANSITIVE GRAPHS UNIQUELY DETERMINED BY THEIR LOCAL STRUCTURE
}

\author{
JOSHUA FRISCH AND OMER TAMUZ
}

\begin{abstract}
We show that the "grandfather graph" has the following property: it is the unique completion to a transitive graph of a large enough finite subgraph of itself.
\end{abstract}

\section{INTRODUCTION}

Transitive graphs "look the same from the point of view of every vertex"; all vertices play the same role in their geometry. Thus they are a natural model for a discrete, homogeneous geometrical space. In this paper we study transitive graphs whose local structure determines their global structure.

Formally, let $\mathcal{G}$ be the set of finite or countably infinite, simple, undirected, locally finite, connected, vertex transitive graphs. Given a $G \in \mathcal{G}$ and an $r \in \mathbb{N}$, a ball of radius $r$ in $G$ is the subgraph induced by all vertices at distance at most $r$ from some vertex in $G$. We say that $G=(V, E) \in \mathcal{G}$ is isolated if it has the following property: there exists an $r \in \mathbb{N}$ large enough so that, if a ball of radius $r$ in some $H \in \mathcal{G}$ is isomorphic to the ball of radius $r$ in $G$, then $H$ is isomorphic to $G$. Intuitively, the structure of the ball of radius $r$ in $G$ determines $G$ uniquely.

Clearly, every finite transitive graph is isolated: one can take $r$ to be the radius of $G$. However, it is not obvious that there are any infinite graphs that have this property. For example, let $G$ be the biinfinite chain (i.e., the Cayley graph of $\mathbb{Z}$ ). Then any ball in $G$ can be completed to a large enough finite chain. In this paper we give an example of an isolated infinite graph, namely Trofimov's grandfather graph [9]. In fact, we give a countable family of such examples.

Note that the grandfather graph is not unimodular, and so cannot locally resemble finite graphs. The novelty is therefore that it can also not locally resemble any other infinite graph. It would be interesting to find an example of a isolated, finite, unimodular graph.

Date: November 25, 2014.

J. Frisch was supported by MIT's Undergraduate Research Opportunities Program. This research was partially conducted at Microsoft Research, New England. 
This question can be formulated as one of finding isolated points in a natural topology on the set of transitive graphs, namely the BenjaminiSchramm topology 2, 4]. A number of interesting questions arise: what is the Cantor-Bendixson rank of this space? Which graphs are left after the isolated points are repeatedly removed? And what generic properties does such a graph have?

These and similar questions have been previously addressed in regard to the related space of marked groups [5, 6]. In particular, Cornulier, Guyot and Pitsch [7] characterize the isolated points in that space. It would be interesting to understand if the (unlabeled) Cayley graphs of these groups are isolated in the space of transitive graphs.

1.1. Acknowledgments. We would like to thank Russell Lyons for helpful discussions.

\section{Formal Definitions AND RESUlts}

2.1. Transitive graphs. Let $G=(V, E)$ be a graph. We will study the set of graphs with the following properties:

- $V$ is finite or countably infinite.

- $G$ is simple and undirected: $E$ is a symmetric relation on $V$.

- $G$ is locally finite: the number of edges incident on each vertex is finite.

- $G$ is connected: there is a path between every pair of vertices.

- $G$ is vertex transitive; we next define this notion.

A graph isomorphism between $G=(V, E)$ and $H=(U, F)$ is a bijection $h: V \rightarrow U$ such that $(u, w) \in E$ if and only if $(h(u), h(w)) \in F$. A graph automorphism is a graph isomorphism from a graph to itself. A graph $G=(V, E)$ is said to be vertex transitive if its automorphism group acts transitively on its vertices. That is, if for every $u, w \in V$ there exists an automorphism $h$ such that $h(u)=w$. The isomorphism class of a transitive graph $G$ is the set of graphs $H$ that are isomorphic to $G$. We denote by $\mathcal{G}$ the set of isomorphism classes of graphs with the properties described above. In this paper, we will, whenever unambiguous, refer to "graph isomorphism classes" simply as "graphs", and likewise simply denote by $G$ the isomorphism class of $G$. We will accordingly write $G=H$ whenever $G$ and $H$ are in the same isomorphism class.

Given $G=(V, E) \in \mathcal{G}$ and $r \in \mathbb{N}$, let $B_{r}(G)=\left(V_{r}, E_{r}\right)$ be the ball of radius $r$ in $G$. This is the finite induced subgraph of $G$ whose vertices $V_{r}$ are all the vertices at distance at most $r$ from some vertex of $G$, and whose edges $E_{r}$ are the edges of $G$ whose vertices are both in $V_{r}$. Since we are concerned with graph isomorphism classes, and since $G$ is 
vertex transitive, it does not matter with which vertex of $G$ we choose to construct $B_{r}(G)$.

2.2. The Benjamin-Schramm topology and isolated points. The Benjamini-Schramm topology [2, 4] on $\mathcal{G}$ is defined by the following metric. Given $G, H \in \mathcal{G}$, let

$$
D(G, H)=\sup \left\{2^{-r}: B_{r}(G)=B_{r}(H)\right\} .
$$

It is straightforward to verify that this is indeed a metric. In fact, this topology is Polish and zero-dimensional. The sets $\mathcal{G}_{d}$ consisting of the graphs with degree $d$ are compact in this topology.

We say that $G \in \mathcal{G}$ is isolated if it is an isolated point in this topology. By the above definition, this means that there exists an $r \in \mathbb{N}$ such that whenever $B_{r}(G)=B_{r}(H)$ then $G=H$. Since $B_{r}(G)=G$ for every finite $G$ and $r$ large enough, it follows immediately that all the finite graphs are isolated.

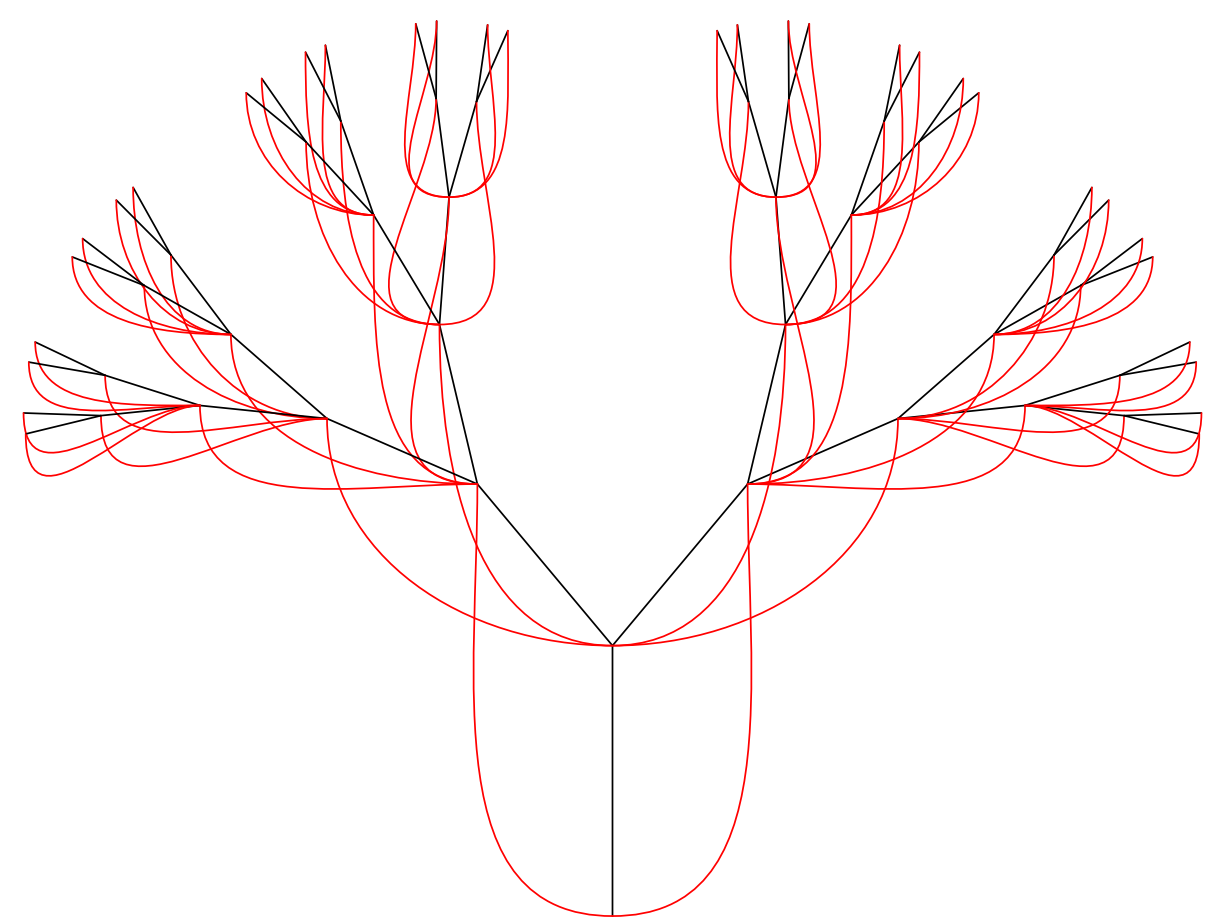

FiguRE 1. The grandfather graph $G_{3}$. Edges of $\mathbb{T}_{3}$ are straight black lines. Edges to grandfathers are red curves. The distinguished end is the "down" direction.

2.3. The grandfather graph. The grandfather graph of order $n \geq 3$, $G_{n}$, is the following graph (see Figure 1 ). Let $\mathbb{T}_{n}$ be the regular tree of degree $n$. The ends of $\mathbb{T}_{n}$ can be identified with the set of infinite 
simple paths starting at $o$, an arbitrary distinguished vertex. Choose a distinguished end. Then each vertex has a unique edge in the direction of this end. Call the vertex on the other side of that edge the "father". Then each vertex has a unique father, and, as one can imagine, each vertex has a unique "grandfather". The set of vertices of $G_{n}$ is identical to that of $\mathbb{T}_{n}$. The set of edges includes the set of edges of $\mathbb{T}_{n}$, and in addition an edge between each vertex and its grandfather.

\subsection{Main result.}

Theorem 1. For $n \geq 3$, the grandfather graph $G_{n}$ is isolated.

In fact, we show below that the ball of radius one determines the structure of $G_{n}$.

We state here without proof that this result can be further extended to some classes of graphs that are similar to $G_{n}$. For example, the product of $G_{n}$ with any finite graph will also be isolated, as will "great ${ }^{k}$ grandfather" graphs.

\section{ProOF}

A directed edge in an undirected graph $G=(V, E)$ is an ordered pair $(u, w)$ of vertices in $G$ such that $(u, w) \in E$.

Let $(u, w)$ and $\left(u^{\prime}, w^{\prime}\right)$ be two directed edges in a graph $G$. We say that $(u, w)$ and $\left(u^{\prime}, w^{\prime}\right)$ are isomorphic if there exists a graph isomorphism of $G$ that maps $u$ to $u^{\prime}$ and $w$ to $w^{\prime}$ (compare to the notion of "doubly rooted graphs" - see, e.g., [1,8]). While all vertices in a transitive graph are isomorphic, not all directed edges are necessarily isomorphic.

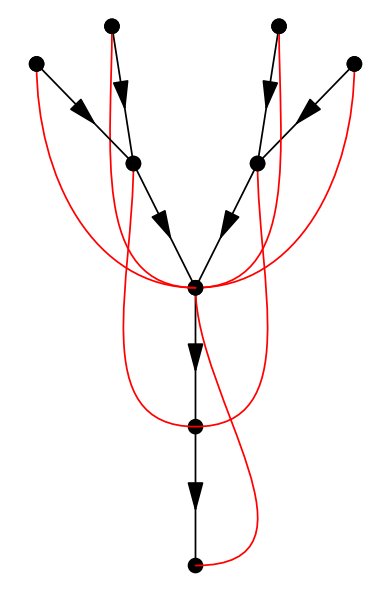

Figure 2. The ball of radius one in the grandfather graph $G_{3}$. The directions and labels of the edges can be inferred from the undirected graph. 
In the grandfather graph $G_{n},(u, w)$ and $\left(u^{\prime}, w^{\prime}\right)$ are isomorphic if and only if both pairs can be described by the same (ordered) familial relation: that is, if $w$ is $u$ 's father (respectively son / grandfather / grandson) and $w^{\prime}$ is $u^{\prime}$ 's father (respectively son / grandfather / grandson). This is a well-known property of this graph (see, e.g., [3, 8]), and is in fact easy to already see by examining the ball of radius one (see Figure 2). In this subgraph, the father and the sons can be distinguished from the grandfather and the grandsons, since their degrees differ. Furthermore, the father can be distinguished from the sons, since the father is connected to the sons (he is their grandfather), while they are not connected to each other. Thus, if $w$ is $u$ 's father but $w^{\prime}$ is not $u^{\prime}$ 's father, there is no graph isomorphism of $G_{n}$ that maps $(u, w)$ to $\left(u^{\prime}, w^{\prime}\right)$.

We can therefore label each directed edge as a father / son / grandfather / grandson edge (that is, $(u, w)$ will be a father edge if $w$ is $u$ 's father), and this labeling will be invariant to any isomorphism of the graph.

This labeling gives rise to an equivalent definition of the grandfather graph: define a father relation on the $n$-regular tree $\mathbb{T}_{n}$; this is any relation in which each node has a unique father which is its neighbor in the graph. Then, connect each node to its grandfather. The choice of a father relation is equivalent to a choice of end, and hence this also results in the grandfather graph.

Let $H$ be any graph in $\mathcal{G}$ such that $B_{1}(H)=B_{1}\left(G_{n}\right)$. Since examining the ball of radius one around each vertex is sufficient to determine this labeling, we can also label the directed edges of $H$ in the same manner, and this labeling will also be invariant to the isomorphism group of $H$. We will use this to show that $H$ is isomorphic to $G_{n}$, which will prove Theorem 1 .

A simple cycle in a graph is a sequence of directed edges $\left(u_{0}, w_{0}\right), \ldots,\left(u_{k-1}, w_{k-1}\right)$ such that $w_{i}=u_{i+1} \bmod k$, and each edge is visited at most once.

Claim 3.1. There are no simple cycles in $H$ which are comprised only of father edges and of son edges.

Proof. Assume by contradiction that $\left(u_{0}, w_{0}\right), \ldots,\left(u_{k-1}, w_{k-1}\right)$ is a simple cycle comprised only of father edges and and son edges. Then all edges are of the same type (i.e., all father edges or all son edges): otherwise, there must be in the cycle a father edge followed by a son edge, which would make the cycle non-simple, since fathers are unique.

By changing the direction of the cycle we can therefore assume without loss of generality that all edges are father edges. Now, note that since $B_{1}(H)=B_{1}\left(G_{n}\right)$, it also follows that every node in $H$ has a unique father and exactly $n-1$ sons. Hence each node on the cycle 
is its own $k^{\text {th }}$-order father, and each node has $n-2>0$ sons which are not on the cycle. Since the father relation is invariant to graph isomorphisms, so is the $k^{\text {th }}$-order father relation.

Let $u$ be a vertex on the cycle, and let $v$ be vertex which is not on the cycle and is a son of $u$. Then there is no graph isomorphism of $H$ that maps $v$ to $u$, since $v$ - unlike $u$ - is not its own $k^{\text {th }}$-order father. Hence $H$ is not transitive, and we have reached a contradiction.

Remark. This claim can also be proved by showing that $H$ is not unimodular and analyzing the Haar measure of the stabilizers of the nodes lying on the cycle (see [8]).

It follows immediately from Claim 3.1 that the restriction of $H$ to father-son edges is isomorphic to $\mathbb{T}_{n}$, the $n$-regular tree. This restriction is still a connected graph, since grandfather edges only connect nodes already connected by length two paths of father edges.

Since $B_{1}(H)=B_{1}\left(G_{n}\right)$, the grandfather edges in $H$ are determined by the father-son relation, and in the same way that they are determined in $G_{n}$. Hence $H$ can be constructed by adding grandfather edges to $\mathbb{T}_{n}$, equipped with a father relation. It follows that $H$ is isomorphic to $G_{n}$, thus proving Theorem 1 .

\section{REFERENCES}

[1] David Aldous and Russell Lyons, Processes on unimodular random networks, Electronic Journal of Probability 12 (2007), no. 54, 1454-1508. MR2354165 (2008m:60012)

[2] David Aldous and J. Michael Steele, The objective method: Probabilistic combinatorial optimization and local weak convergence, Probability on Discrete Structures (Volume 110 of Encyclopaedia of Mathematical Sciences), ed. H. Kesten 110 (2003), 1-72.

[3] Itai Benjamini and Nicolas Curien, Ergodic theory on stationary random graphs, Electron. J. Probab 17 (2012), no. 93, 1-20.

[4] Itai Benjamini and Oded Schramm, Recurrence of distributional limits of finite planar graphs, Electronic Journal of Probability (2001), 1-13.

[5] Christophe Champetier, L'espace des groupes de type fini, Topology 39 (2000), no. 4, 657-680.

[6] Yves Cornulier, On the cantor-bendixson rank of metabelian groups, arXiv preprint arXiv:0904.4230 (2009).

[7] Yves Cornulier, Luc Guyot, and Wolfgang Pitsch, On the isolated points in the space of groups, Journal of Algebra 307 (2007), no. 1, 254-277.

[8] Russell Lyons and Yuval Peres, Probability on tress and networks, 2013.

[9] Vladimir Trofimov, Automorphism groups of graphs as topological groups, Matematicheskie Zametki 38 (1985), no. 3, 378-385.

Department of Mathematics, Massachusetts Institute of Technology, CAMBRIDGE MA 02139, USA. 